\title{
THE TYPHOON DISASTER ANALYSIS EMERGENCY RESPONSE SYSTEM BASED ON UAV REMOTE SENSING TECHNOLOGY
}

\author{
Chen Qiujin ${ }^{1,2}$, He Yuanrong ${ }^{1,2}, \mathrm{He}_{\text {Tingting }}^{3}, \mathrm{Fu}_{\text {Wenjie }}^{2}$ \\ ${ }^{1}$ College of Computer and Information Engineering, Xiamen University of Technology, Xiamen 361024, China- \\ cqj0818@outlook.com,2012112001@xmut.edu.cn \\ ${ }^{2}$ Key Laboratory of Ecological Environment and Information Atlas (Putian University) Fujian Provincial University \\ ${ }^{3}$ The Civil Engineering And Architectural Association of Fujian,Fujian Xiamen 361022, China
}

KEY WORDS: UAV remote sensing, typhoon disaster emergency system, Web GIS, disaster analysis, ArcGIS Server

\begin{abstract}
:
The satellite image data has some shortcomings such as poor timeless, incomplete disaster information and so on in the typhoon disaster analysis. Compared with the satellite image data, unmanned aerial vehicle (UAV) remote sensing technology has the characteristics of flexibility, convenience, high resolution and so on. It plays a great role in the aspect of obtaining the images and systematically analyze the disaster data. This research based on UAV technology to obtain the high resolution image data and complied the disaster thematic maps after interpretation, as well as determining the data model. Subsequently, determing the system used Html, Javascript and CSS to build the system framework. Combining with Postgre SQL database, Leaflet map module and Echarts diagram and other technologies to perform the feasibility analysis and the detailed design of the integrated system. Finally, it could accurately and comprehensively obtain the system's disaster monitoring, the typhoon track display, the diagram statistics and visual analysis of the data processing, as well it could deeply analysis and management for the disaster information and assessment. The application shows that this system could provide the information support for future emergency rescue, which is of great significance for the monitoring and preventing the occurrence natural disasters in the future.
\end{abstract}

\section{Introduction}

China has a wide length and breadth of land, the geographical environment is intricate, and the natural disasters occur frequently.China is one of the countries which was most severely damaged by natural disasters in the world(Wang et al.2008a).The frequent occurrence of natural disasters such as typhoon brings a great threat of the safety to people's lives and property in coastal areas. The disasters had caused many influences such as interrupting the communication-equipment and destructing the transportation infrastructure.It is hard to obtain the real-time dynamic information in the disaster-affected area by routine measures,and it can not effectively and comprehensively understand the loss situation in time. Thus affecting the follow-up rescue work(Ding,2012b). Due to the long re-entry cycle of satellite aerial images, the high price of the image, the high cost of use and so on. As well as the manned aerial remote sensing was restricted by air traffic control. At the same time,the monitoring and the loss assessment after typhoon are the important basis for the scientific decision-making of disaster preventing and mitigation(Fan et al.2016a).Therefore,UAV remote sensing technology has became an indispensable monitoring mean after the disaster. Based on the existing typhoon disaster remote sensing image data and research findings, the typhoon emergency response system was designed to prevent and respond to the typhoon disasters integrated disaster data information in each region,which was based on the work foundation and data results accumulated by the unmanned aerial vehicle emergency surveying and mapping carried out after the typhoon "Meranti", thus we could further visualize and analyze the disaster data.

At present, the research of typhoon disaster has became the focus object of many domestic and foreign experts and
scholars(Lu et al.2011a).The research objects are mainly based on the the national and provincial large areas. The research content is mainly concentrated on the areas of the typhoon prediction and the post-disaster monitoring.In the foreign countries,international research as early as 1991,Emanuel et al. (Emanuel et al.1991b) applied the unmanned aerial vehicle to tropical hurricane observation. In 2006,Pratt et al.(Pratt et al.2006a) used a helicopter UAV equipped with a digital camera to explore the effects of UAV in the investigation of the damage to the multi-storey commercial building after Hurricane Katrina,and they pointed out that UAV and other technologies have great potential for data acquisition and disaster assessment after the natural disasters. In 2011, Chen and others(Chen et al.2011a) built an integrated platform in order to realize the timely release of kinds of weather information. The platform includes the weather forecast, the typhoon real-time path information, the meteorological satellite cloud image and the radar image. In 2012, in order to improve the rapid response of disaster and timely processing of fallen trees,Szoltan et al.(Szoltan et al.2012a) developed a tool to rapidly detect the fallen trees by using Leica digital aerial sensors and high-precision data images. The tool was used to count the damage of trees in the urban areas under the influence of hurricanes.In China,Chinese scholars have also made some outstanding contributions in this research field. In 2011, Zheng Xiaoyang et al.(Zheng et al,2011a) collected all typhoon information and monitoring data in Shanghai since 1999, and on the basis of a large number of data, they built a typhoon information service system based on WebGIS including a route module,an early warning module,a measurement and calculation module,a query and statistic module,and an influence radius analysis module.In 2014,Wang Yan et al.(Wang et al.2014a)discussed the destruction of the coastal zone of typhoon landfall at Wenchang in Hainan province after the 
typhoon "Weimaxun" was used as the investigation and monitoring basis. They introduced the composition and the advantages of UAV remote sensing systems, as well as the monitoring process of data acquisition and processing management based on UAV remote sensing technology. Combining with the remote sensing data,In the same year,Zhang Guangping et al.(Zhang,Gao,2014a) used the WebGIS technology to build a command and management system that coped with the typhoon disasters. The system achieved the typhoon pre-warning and forecasting, the real-time monitoring in the disasters, the statistics of the post-disaster losses and integrated the emergency rescue team management, the windproof material management,the plan management, the work situation management, the disaster statistics management and other business services.The system has also realized integrated management of the typhoon disasters.

In summary, the research on typhoon disasters in China and abroad is mainly based on the satellite data.The current remote sensing technologies most apply to the typhoon monitoring and the impact assessment after disaster.As one of the countries most affected by typhoon disasters, in the aspect of the prediction and preventing typhoons, China's government and related departments are mature in research application.However, there are few reports on the typhoon disaster loss assessment, the post-disaster recovery and the daily urban management etc. based on the UAV technology.Therefore, this research is based on the UAV image data with high resolution to analyze the disaster situations and construct the emergency response system.This paper was based on the centimeter-level orthophotos acquired by the UAV remote sensing system and established the UAV image interpretation marks of the affected bodies. This paper looked up the data information about typhoon disaster over the years and realized the data integrated management, the process processing and the visual mapping outputting by the system development.The results of this research could meet the typhoon's emergency response capability and provide a fast,accurate and transparent integrated technology platform for the planing and management of urban projects after disaster,which is very meaningful for monitoring and preventing the occurrence of natural disasters in the future.

\section{THE GOAL AND KEY TECHNOLOGIES OF THE SYSTEM}

\subsection{The overall goal}

At present, the most typhoon research systems are based on the satellite image to analyze the typhoon disaster situation, it leads to some research status such as the weak timeliness, the insufficient analysis methods, the inadequate system content of disaster information and the system integration development and application should be excavated.This research is mainly aimed at the requirements of typhoon disaster analysis and monitoring service from the government and the related institutions.Combined with PostgreSQL database,Html architecture,Tomcat and ArcGIS Server server,and by integrating the application spacial analysis, the mobile GIS and WebGIS and other related technologies to obtain the high-resolution image data visualization analysis and application development what acquired by the UAV remote sensing,so that it could meet the emergency response ability of typhoon and provide the fast,accurate and transparent integrated technology platform for the planing and management of the urban repairing projects after disaster.

\subsection{The key technologies}

This system adopts the three key technologies, WebGIS technology,JavaScript and PostgreSQL database to use the high-resolution image data what were acquired by the UAV remote technology to monitor the typhoon disaster,data processing management and analysis as well as disaster visual display.

(1) Web GIS technology:

The HTTP standard application system protocol is embedded in the traditional GIS to realize the sharing and interoperability for the geospatial information and the data resources among all fields and departments of the whole society. The client uses a web browser and the server provides the information and services to the client. WebGIS could not only realize the functions of traditional GIS, but also realize the functions of data acquisition, storage, processing, collation, management analysis and visualization in the geographic information. Recently the functions of WebGIS application include the following aspects(Li,2016b): (1)Map visualization and query function: It could realize the query and retrieval of the graphics and attribute data, as well as enable clients in different region also could operate these data by interacting with browser.(2)Geographic information dissemination: Using a general web browser, users could access the Web GIS data transparently and quickly. It could process and analyze the distributed multi-source data on the server, and the sharing of geographic information data can be realized, and the data resources can be effectively used to avoid data duplicating collection and waste of resources.(3)Geospatial information analysis:Providing kinds of analysis and methods of various application models on the high-performance server side. Carrying out the calculation and analysis quickly by receiving the model parameters what were provided by the users. The calculation results are returned to the browser in a timely manner, such as graphics or text.

(2) JavaScript:

It is a lightweight programming language, it's also a network scripting language. The source code doesn't need to be compiled before sending it to the client. Instead, it sends the character code in the text format to the browser for interpretation and operation by the browser. JavaScript has been widely used in web application development, it commonly used to add a variety of dynamic functions for web pages. It could provide the more smooth and beautiful browse effect for users.JavaScript usually realize their own functions by embedding them in HTML, such as responding the users' requests, animation interaction effects, and so forth.

(3) PostgreSQL:

PostgreSQL almost supports all types interfaces of database client. The database server provides a unified client $\mathrm{C}$ interface.Different client interfaces are derived from this $\mathrm{C}$ interface,such as ODBC, JDBC, Python, Perl, Tcl, C/C++, ESQL,etc(Yang,Xu,2011a). PostgreSQL database provides a logical, strict structure, data integrity and supports the multi-user concurrent background relationships for the client platform by designing the data storage management and the data retrieval models. PostgreSQL uses GNU Readline for interactive SQL queries, and it uses a multi-row data storage strategy called MVCC to enable PostgreSQL global access in high-capacity environments, and it could realize high-capacity and high-performance data access.

\section{THE ARCHITECTURE AND DESIGN OF THE SYSTEM}


Based on the deep understanding of the application status of the typhoon emergency response integrated system and WebGIS technology,and considering the actual situation to perform the research analysis for the database construction of the typhoon emergency response integrated system.Determing the development architecture,the network function module and the system map structure etc. what are suitable for the typhoon emergency response integrated system with the support of the related technologies.The cloud server with PostgreSQL database in the sytem's background is used to store the typhoon information data and provide the data source for the system clients. The client chooses HTML,JavaScript and CSS language to build the front end,and combined with WebGIS technology to develope the server on the Tomcat and ArcGIS Server.According to the system design requirements and feasibility analysis to propose the overall design structure of the typhoon emergency response integrated system.This system adopts the $\mathrm{B} / \mathrm{S}$ architecture(Browser/Server), which is divided into three layers frameworks system:the data service layer,the logic service layer and the presentation layer.The logic service layer could divided into the Web service layer and the profession logic layer(Dai,2009a). The architecture is shown in Fig.1.

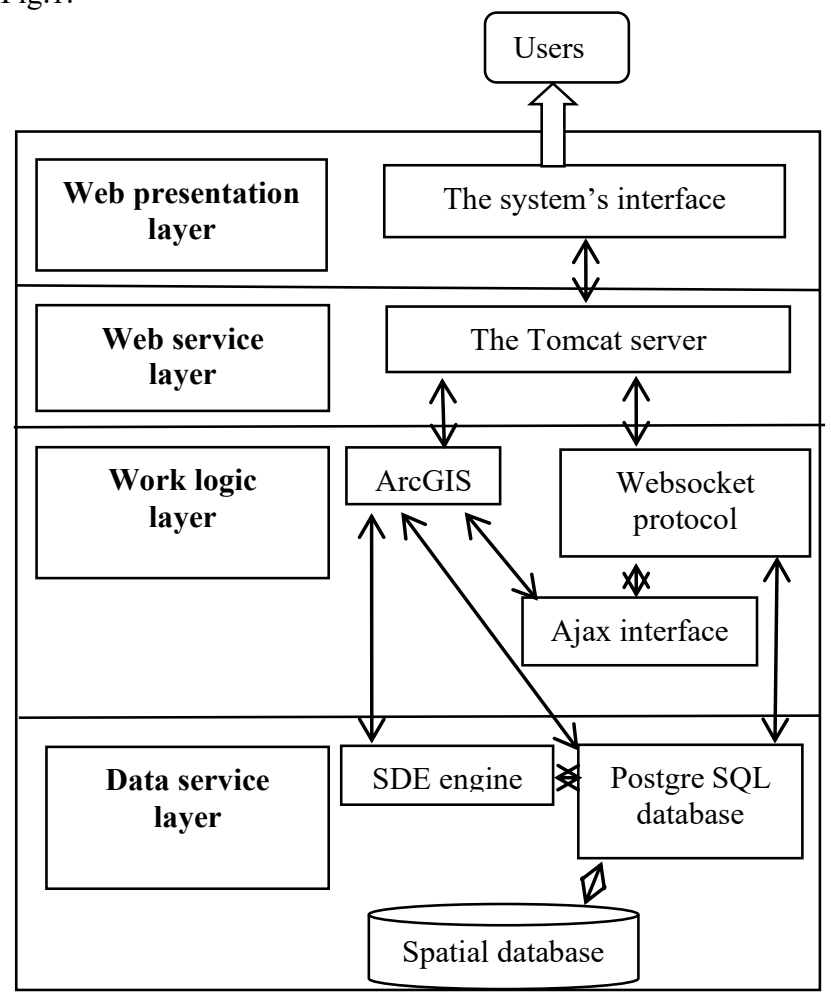

Figure 1.The system's overall architecture

(1)Data service layer:This layer includes the typhoon information database and the spatial database. The data from PostgreSQL database provide the map data source for the system through by ArcSDE engine and the ArcGIS Server. This layer mainly stores the system's spatial map data and non-spatial attribute data.

(2)Work logic layer:The server object and map data interfaces are obtained from the ArcGIS Server, so that this layer provided a spatial Web services for the use of Tomcat server.

(3)Web service layer: The typhoon emergency response system used Tomcat as the Web server in this service layer.

(4)Web presentation layer:This layer, namely client, has shown the content of map data by the browser(Chen,2018a).So that users could use some functions such as browsing, querying typhoon information and the remote sensing image map data of "Meranti" typhoon, map magnifying or minifying and so forth.

\subsection{The functions and compositions}

This system is aimed at the research status, which the improvement of the resolution of the UAV remote sensing in the order of magnitude,but the analysis method of disaster information is not deep enough, and the potential of the system integrated development and application is still to be excavated.The investigation and analysis of the serious damage in the research area which was against the typhoon "Meranti".This typhoon emergency response integrated system mainly includes the typhoon data management,the disaster data management,the disaster thematic management, the thematic map visualization,the report output statistics and the data visualization analysis. The functional modules of this system are shown in Fig.2.

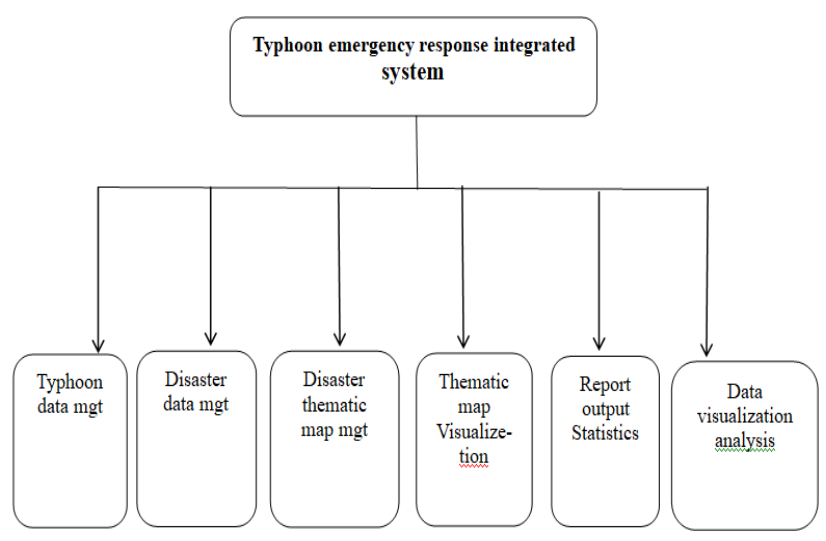

Figure2. The functional module diagrams of the system

(1)Typhoon data management:Obtaining the typhoon information data (the typhoon occurrence time, the latitude and longitude, the typhoon location, the typhoon's route path, the typhoon wind power and wind speed or other information) to store in the PostgreSQL database over the years from 1945 to 2018.Combined the PostgreSQL database typhoon information data of the system's backend with the geographical information, and according to the query conditions of the response to retrieve the each data in the database. And the development trend of kinds of typhoons is displayed in the form of a line graph or a chart.

(2)Disaster thematic map management: According to the centimeter-level aerial images after the typhoon disaster which were acquired by utilizing the UAV aerial photography technology. In order to achieve the upload management services of the disaster thematic map which is based on the ArcGIS server so did the comprehensive interpretation of the disaster information, the systematic in-depth analysis and statistics, as well as the standardized cartography and geographical registration with the function "offset correction" of "China Map Color Edition" in the ArcGIS map service.

(3)Disaster data management: The disaster data is mainly based on the analysis of the UAV images after the "Meranti" typhoon disaster in Jimei District. And establishing the UAV images interpretation marks of the affected bodies at the disaster district such as trees, street lamps, poles, houses and 
water-storage area etc. As well as performing the spatial analysis, statistical analysis and image information extraction for the detailed disaster data. Then the classification management of the variety of types of statistical ground objects information which calculated by the disaster thematic map is stored in the server PostgreSQL database.

(4)The thematic map visualization:According to the site survey to understand the type of damage and establish the interpretation marks, and drawing the corresponding disaster thematic maps to analyze and visually express the geospatial data(DavidArctur et al.1998a). In this system, the damaged ground objects and the attribute data after the typhoon disaster were presented in the form of interpretation marks in the thematic map, and they were expressed in an intuitive form on the map.

(5)Reports output statistics:The reports have became an effective way to display and statistic data in information systems. All typhoon data in the database can be obtained according to the users' query conditions(Disaster-affected communities and disaster elements), and the results are output to the corresponding reports(Huang,2012b)(in the format of Excel or txt). In addition, after the report was outputted, it can also be set the print effect. And the relevant departments can browse and look into the typhoon damage information more intuitively and specifically.

(6)Data visualization analysis: The data visualization refers to displaying data with the forms of a histogram, a pie chart or a line chart,etc.It could help the analysts have a more comprehensive understanding of data and more convenient analysis of the data.This function not only provide the display function of the typhoon information data with hourly interval,but also it could perform the thermal map analysis for the typhoon path to more intuitively analyze the typhoon route path and the changing characteristics of the trend.

\subsection{The structure design}

3.3.1 The design of the map layer : Leaflet is the leading open source JavaScript library for building moving and friendly interactive maps. It provides users with most online map development functions(Zhang,2015b).Leaflet follows the principles of simplicity, high performance and availability in development and design, and operates efficiently.It could intensify the expansibility of the system by taking advantage of HTML5 and CSS3 in browsers. Creating a map in the system framework, that is,the HTML file with the 'div id="map" $></$ div' statements, adding the map of our choice, and then adding some identifiers with text in the popup windows. The implementation steps of the execution code as follows:

1)Configuring the initialization of the leaflet's base year.var baseLayer $=$

L.tileLayer('http://\{s\}.tile.openstreetmap.org/ $\{\mathrm{z}\} /\{\mathrm{x}\} /\{\mathrm{y}\}$.png'); 2)Loading the typhoon vector data layer.

var shplayer $=$ L.esri.tiledMapLayer $(\{$

url:'http://172.16.240.33:6080/arcgis/rest/services/Typhoon/TFs

huju7/MapServer', \});

3)Loading the typhoon image layer.

var imalayer $=$ L.esri.tiledMapLayer $\{\{$

url:'http://172.16.240.33:6080/arcgis/rest/services/Image/TFyin gxiang6/MapServer', \});

4)Defining the typhoon point and the line data heat level var pointOverlayer $=$ L.layerGroup(); //typhoon point layer var lineOverlayer = L.layerGroup(); //typhoon line layer

5)Initializing the setting of the map center position and the zoom level. var map = new L.Map('mapContainer', \{center: new L.LatLng(24.65,118), zoom: 13, layers: [baseLayer, imalayer, shplayer, lineOverlayer, pointOverlayer] \});

3.3.2 The design of database:In the part of database design of this system,the combination of ArcSDE and Postgre SQL database is used to store the typhoon information data. The typhoon information data is further divided into the spatial data and the attribute data. The spatial data is mainly the remote sensing maps acquired by the UAV and the interpreting data of the affected bodies, and the attribute data is mainly typhoon information data over the years. This system established a unified multi-functional platform for data management,processing analysis and reporting statistics.After deploying the server database, this system designed to store the all typhoon information data and the disaster thematic map of Jimei District and other affected bodies over the years.After the "Meranti" typhoon, the high precision images were obtained by the aerial photography in the Jimei District of Xiamen which were based on the UAV technology, and the site surveys and demand analysis were carried out.Establishing the interpretation marks data table in the server to perform the data management for the disaster data after the typhoon disaster.

\section{THE DEVELOPMENT AND IMPLEMENTATION OF THE SYSTEM}

This typhoon emergency response integrated system, which is as a subsystem of the Digital Natural Disaster Monitoring Big Data System in Fujian province, is mainly divided into three modules: the map module,the diagram module and the report outputting module. These three modules not only include the basic map operation functions of WebGIS, but also the display of the typhoon route paths, as well as using ECharts controls and other tools to realize the display of the typhoon information and the function of the report data statistics. This system established a unified multi-functional platform for data management, process analysis and reporting statistics and so forth.

\subsection{The map module}

At present,the typhoon information of meteorological observation contains many contents, which includes the typhoon name,the typhoon wind power,the wind speed,the center position,the center air pressure,the radius of seven-level or ten-level wind circle,the positioning time,the moving direction and speed,the forecast of 24-hour and 48-hour,the forecast meteorological station and other functions(Luo,2008b).This system realized the display function of the map release and typhoon information on this function. It could be clearly understood the disaster data of the four districts of Xiamen, Jimei, Xiang'an, Tongan and Siming after the typhoon "Meranti" passed in the map module. In the interface of the system,we could drag the mouse to move the map and zoom in or out in order to observe and analyze the damage situation in each area in more detail. The icon in the upper right corner of the map could be clicked to select the main typhoon information collected over the years, the icon in the lower left corner could be show the legends of kinds of affected bodies, and the main function to realize the data visualization analysis in the lower right corner. As shown in Fig.3, the main main interface of the system (the map function module) shows the related typhoon information:

4.1.1 The operation of the map:The implementation of the map's basic operation includes:magnifying,narrowing, roaming 
and the display function of legend.It is mainly for users to operate the map more conveniently and quickly,which could reach to the function of comprehensively obtaining the map relevant information.(1)The function of magnifying and narrowing the map.After selecting the typhoon,the system will automatically generate the typhoon information in the world map what was provided by OpenStreetMap.We can zoom in or out the function key or by rolling the mouse button to realize the function of magnifying and narrowing the map. (2)The roaming function of the map.It could drag the map to realize the function and effect of the map roaming.(3)The function of the legend display.The function button of a legend in this system interface, clicking this button could display the various of legend information of the affected which are from the uploaded "Meranti" typhoon disaster remote sensing thematic map.By comparing the legend and the thematic map,it could clearly reflect the information expressed by the affected bodies. bodies on any typhoon in the upper right corner could display the details of the typhoon (including the typhoon name, the wind force, the wind speed, the center position, the center pressure, the seven-level or ten-level wind circle radius, the positioning time, the moving direction and speed,24 hours and 48 hours forecasts, forecast weather station, etc). The figure 3 shows the detailed information of typhoon "Meranti" in 2016 and shows its propagation path in the form of a heat map:

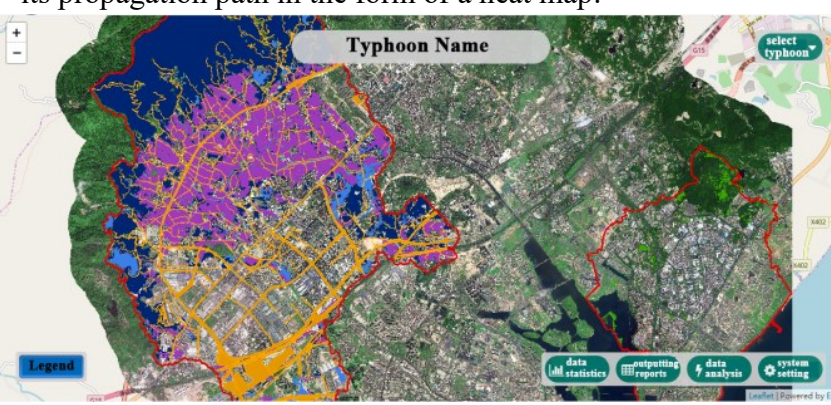

Figure 3. The main interface map module of the system

4.1.2 The display of the information: When the typhoon occurs, the government department and the related departments are most concerned about the typhoon moved trail and passed region,as well as includes the typhoon wind power,the wind speed,the central position,the central pressure,the moving direction and speed and other typhoon information. Therefore making the corresponding rescue disaster plan and the preventive measurement.

The typhoon information display of this system could be query according to the year of typhoon occurrence.In the information query interface,the system will sort out the time and the name information of all typhoons according to the chronological order.The users could select the typhoon what is interest,the client will display the statistical diagram and the statistical table according to the selected typhoon. The typhoon statistical diagram shows the start and the end time ,the wind speed and the wind power information of the typhoon.It could move the mouse in the statistical diagram, when the mouse move to the random point position, it would display the wind speed and the wind power and other data information of this typhoon of this moment.The typhoon statistical table shows the latitude and longitude of the typhoon center as well as the wind power and the wind speed information so that the related departments could effectively estimate the typhoon damaged areas' loss.It can also display detailed information of any typhoon(including typhoon name,wind power,wind speed,central position,central pressure,wind circle radius of seven-level and ten-level,positioning time,moving direction and speed,forecast of 24-hour and 48-hour,forecast meteorological station,etc ).As shown in Fig. 4 is the detailed information of typhoon and the thermal map of the 2016 "Meranti" typhoon what was shown its propagation path.

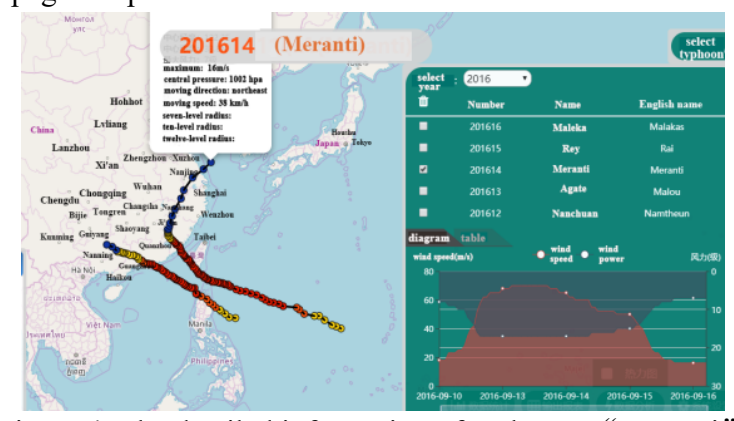

Figure 4. The detailed information of typhoon "Meranti"

\subsection{The diagram module}

4.2.1 The query of diagram:ECharts is a data visualization chart library based on JavaScript that provides intuitive, interactive and customizable data visualization charts.Its underlying layer is based on a lightweight vector graphics rendering library that provides an intuitive and interactive rich, highly customizable data visualization chart(Xiang,2013b).ECharts provides the regular line charts, the histograms, the scatter plots, the pie charts, the K-line charts, the block diagrams for statistic, the maps, the thermal maps, the line graphs for visualization of geographic data for displaying the relational data and the parallel coordinate map of multidimensional data visualization. By calling to the ECharts control, the system has implemented the detailed display functions of the typhoon information in the form of charts or graphs. In selecting the typhoon display function, the top half of the typhoon selection chart arranges the typhoon information according to the chronological order of occurrence and makes the typhoon information were clearly displayed to users in each time period. However, in the lower half of the typhoon selection chart, the typhoon was selected by the user and the system could display the specific typhoon information by the statistics and charts which were provided by the system. The statistical line chart shows the wind speed and the wind power size information from the start to the end of the typhoon. Similarly, the data table shows the typhoon name, the latitude and longitude, the wind force, and the wind speed information in the form of a table.As shown in Fig.5, the diagram module of this system shows the statistical diagram and the data table of the typhoon "Meranti" in 2016.

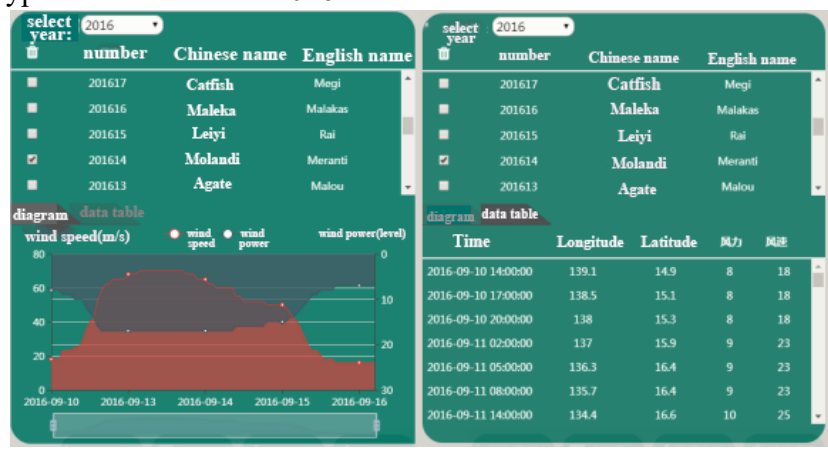

Figure 5.Typhoon information statistical diagram, data table

4.2.2 The implementation of diagram:The users could choose one of the damaged area signs such as the dumping poles,the fallen trees, the fallen street lights, the stagnant water areas, the damaged farmland,the damaged parkingspaces, the damaged houses areas, the damaged areas of factory buildings, the tree 
lodging areas, the damaged traffic sites, the damaged garden land the damaged areas of iron house. The system could display the statistical results in the form of a default histogram by a statistical querying.In the upper right corner of the data statistical interface,the user could switch to a line chart for displaying according to user's need. It can also use the area zoom function key to individually select and analyze the data of

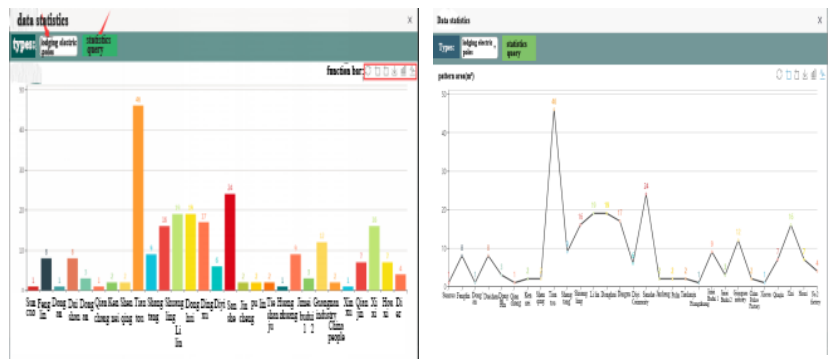

function of the statistical module (save picture format is PNG) to compare with other data.For example, the data statistical histogram and line charts of the lodging electric poles are shown in Fig.6 and Fig.7, respectively:

Figure 6 . The data statistics histogram of lodging electric poles Figure 7. The line graph interface of the lodging electric poles

\subsection{The report outputting module}

4.3.1 The process of technology:The system can generate the reports of the typhoon disaster information in order to realize the analysis and release of data information. The report is an important reference for the management of the typhoon projects. The information data it provides has a positive effect on the government and the related department in post-disaster restoration:It not only could comprehensively and systematically reveal the disaster situation and the degree of damage about the characteristic geographical things within a certain period time in the affected area, which could provide the accurate statistical data for the superior departments timely and accurately.The report also has the characteristics of high recovery rate, flexible mode and so on.The management personnel can form the time series data of the typhoon's reports over the years, which is convenient for the relevant departments to provide the horizontal and vertical reference.

The report function module is divided into the three layers, the middle layer is divided into the three steps: the request listener, the data acquisition and the report outputting. The report listener feeds back the users' choice to the database; The data acquisition includes several steps. First, it is necessary to connect the database in order to query the data set required by the system user from the database, and the data set is added to the report; The report outputting only requires the users to select the desired format: Excel or TXT report outputting. The workflow of the entire report modules is shown in Fig.8:

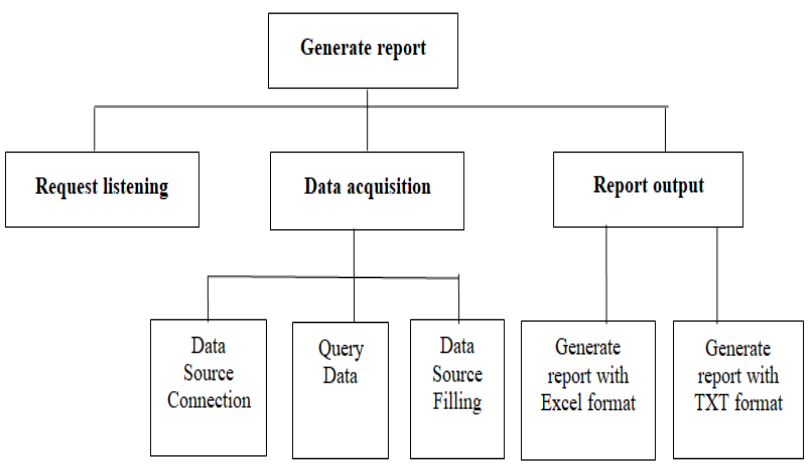

Figure 8. The report outputting module process tree
This system could provide the flexible function of report screening to support the analysis and release of typhoon disaster data information. The information report and the outputting set two information selection modes, the one is output and report the all data from a database,the other mode is entering the form of data screening for query and selecting the outputting and report.In the data report function module interface, the users could select the name of the department and the category of the elements according to his demands. And the server will request the database and feed it back to the client. The system will display the affected body information in the remote sensing thematic maps such as the name of the related department and the ground categories, the spot area and the number of the spots. The data report module for all affected bodies is shown in Fig.9.

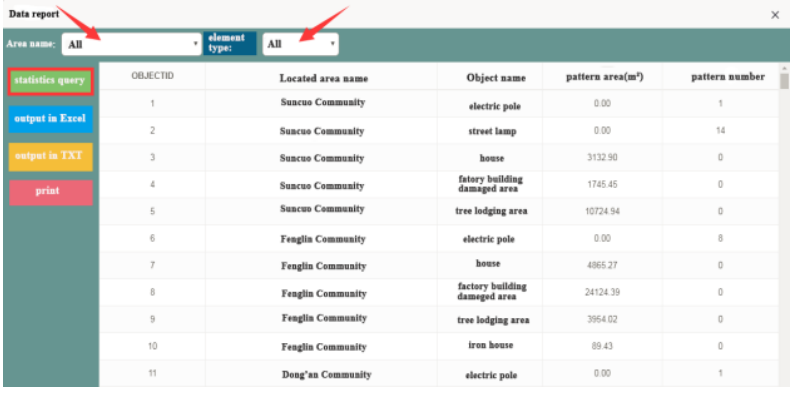

Figure 9. The data report function module of the all affected bodies

4.3.2 The export of process:This system could timely output the query results according to the demand,and provide the format with TXT and Excel to output and print the data,which could provide the multi-channel data viewing platform for users. There are two ways to export and print the report chart: (1) By the browser's print menu. The browser's own printing function can set the printing direction, the margins, adding headers and footer of printed pages and so on. (2) Printing after exporting the file. It mainly in the format of PDF, it could read the PDF files in the web browser of Acrobat Reader plugin.After the application opens a PDF report, we could use the application's own print function to print preview and precise print settings.In a word,it is convenient and direct to use the print function of the browser to realize the printing service of report. It also could add the information such as time,page number,title,URL and so on,which is easy to display and save the diagram results.

\section{CONCLUSION}

This research made use of python technology to obtain the typhoon data information.And taking advantage of the UAV remote sensing technology to obtain the remote sensing orthophoto images of "Meranti" typhoon after disasters, then interpreted the images and draw the corresponding disaster thematic maps. The logical model of the database was determined according to the correlation between the typhoon's grid and vector data.Subsequently, designed the overall framework of the typhoon emergency response integrated system which was based on the OpenStreetMap world map, as well as we completed the detailed design of the system; Finally, the users could look over the typhoon information at any time in the system and it could be performing the diagram statistics and visual analysis display.The application result shows that this system provides the users with concise, accurate, comprehensive typhoon data over the years and the disaster thematic maps, and provides the information support for 
emergency rescue in the future. Even more doing a good job in the emergency mapping for the key areas and the map information data resource reserves in order to deal with the typhoon approaching.It could also meet the capability of typhoon emergency response, which is of great significance for future monitoring and prevent the occurrence of natural disasters.Meanwhile, the typhoon emergency response system still exists some shortcomings, such as the system data transmission is slow,the functions is not perfect and so on.In the future,for the problem about the data transmission is low,we could optimize the operation efficiency of database.In the aspect of expanding the system functions, we could expand the typhoon radar map,the cloud map and other spatial attribute function, as well as intensifying the data analysis ability and so on.

\section{ACKNOWLEDGEMENTS}

This research was supported by the Science and Technology Planning Project of Xiamen/Quanzhou City (grant no. 3502Z20183055, 2017G030);

and the Open Fund Programs of Key Laboratory of Ecological Environment and Information Atlas (Putian Universi ty) Fujian Provincial University(Grant No.ST17002).

\section{REFERENCES}

Wang Jun, Xu Shiyuan, Shi Chun, et al. Dynamic Assessment of Typhoon Disaster Based on Multi-source Remote Sensing Image:Research Progress [J]. Journal of Natural Disasters, 2008, 17(3): 22-28.

Ding Xiaoming. Research and design of tunnel lighting control system [D]. Xi'an: Chang'an University, 2012.

Fan Yida, Wu Wei, Wang Wei, et al. The research progress in disaster remote sensing in China [J].Journal of Remote Sensing, 2016,20 (05): 1170-1184.

Lu Bodi, Meng Diwen, Lu Ming, et al. Application and Discussion of UAV in the Major Natural Disasters [J].Disaster Science, 2011,

Emanuel K ,Anderson J, Hurricane reconnaissance by pilotless aircraft; proceedings of the Proc $19^{\text {th }}$ Conf on Hurricanes and Tropical Meteorology, Miami,Amer Meteor Soc,F[C],1991.

Pratt K, Murphy R, Stover S,Griffin C.Requirements for semi-autonomous flight in miniature uavs for structural inspection[J].AUVSI's Unmanned Systems North America Orlando,Florida, Association for Unmanned Vehicle Systems Intemational .2006.

Chen Z,Bai J.The Design of Typoon Meteorological Information System and its Implementation Based

WebGIS[J].Procedia Environmental Sciencs.2011.10(Part

A):420-426

Szoltan Z,Malone S,Escobedo F,Misas O,Smith S,Dewitt B. A tool for rapid post-hurricane urban tree debris estimates using high resolution aerial imagery[J].International Journal of Applied Earth Observation \& Geoinformation, 2012,18(1):548-556.

Zheng Xiaoyang, Gao Fangqin. The Reseach on the Typhoon Information Service System Based on WebGIS[J].Urban Roads Bridges and Flood Control, 2006(1):39-42.
Wang Yan, Hong Hailing, Wang Pei, et al. The Application of UAV remote sensing in typhoon disaster investigation[J]. Ocean Development and Management, 2015, 32(12): 60-63.

Zhang Guangping, Xie Zhong, Luo Xiangang, et al. The typhoon disaster management decision aid system based on WebGIS in Hainan Province[J].Journal of Tropical Oceanography,2014,33(6):80-87.

Li Yuanyuan.Design and Implementation of Qinghai-Tibet Highway Permafrost Disaster Information System Based on WebGIS[D]. Lanzhou: Lanzhou University, 2016.

Yang Zheng, $\mathrm{Xu}$ Xiaobiao. Design and Development of the Dynamic Web Page Templates Based on Web Database[J]. National Land Resources Informatization, 2011(2):44-46.

Dai Wei. Research on the Application of WebGIS Typhoon Prediction System Based on ArcGIS Server[D]. Wuhan: Wuhan University of Technology, 2009.

Chen Heng. The Tourism Website Development and Implementation based on Web Technology [J].Modern electronic technology, 2018, 11 (7): 34-36.

DavidArctur, DavidHair, GeorgeTimson, et al. Issues and prospects for the next generation of the spatial data transfer standard (SDTS)[J].International Journal of Geographical Information Systems, 1998, 12(4):403-425.

Huang Liting. Research and implementation of ontology-based semantic reporting system [D].Beijing: Beijing University of Posts and Telecommunications, 2012.

Zhang Wenjun. Front-end design and implementation of flash flood warning and deduction system based on AngularJS[D]. Wuhan: Huazhong University of Science and Technology, 2015.

Luo Qingwen.Design and implementation of early warning information release system for major rice diseases and insect pests in Shanghai based on WebGIS[D].Shanghai:Shanghai Jiaotong University, 2008.

Xiang Yunlu. Research and Implementation of the Typhoon Information Retrieval System Based on WebGIS[D].Wuhan: Wuhan Polytechnic University, 2013 